\title{
COGNITIVE GRAMMAR AND MAP DIGITIZATION
}

\author{
Eero Carlson \\ National Land Survey of Finland \\ P.O. Box 84 \\ FIN-00521 Helsinki, Finland \\ carlsonemmh.mailnet.fi
}

The new conception of natural language structure introduced by Ronald Langacker is applied to graphic language. Symbolic structures reside in the relationship between a semantic and a phonological or graphical structure. The usage-based nature and the content requirement of the cognitive grammar corresponds to the self-organizing systems, where usage events are categorized and form regions in the given domains.

Meaningful structures are learned from examples and clustered around prototypes. The structure at a given level is elaborated by substructures. Primary and secondary participant - termed the trajector and the landmark - motivates the categorization of the usage events and the recognition of the figures from the ground.

Two or more component structures are integrated to form a composite structure. Components are categorized by the component Kohonen map and their relationships by the composite Kohonen map. Salient objects described as trajector/landmark pairs focus the attention to the entities with maximal meaning and other components can be obligatory or optional.

Map digitization consists of three phases. Firstly old originals are scanned, secondly raster data is vectorized to graphemes and thirdly the meanings of the symbolic structures are given or recognized.

In the test environment graphemes are formed as one to five vectors. These graphemes are self-organized on the component Kohonen map. Composite structures consist of one to five trajector/landmark pairs and are organized on the composite Kohonen map.

The recognition of the handwritten character "2" ('the bench under the tree') is tested and the recognition rate of over $90 \%$ is achieved, depending on the chosen distance to the prototype. If all characters are recognized, then many wrong characters, which have the same components $(8,3,5$ and others) are also recognized and the ambiguities should be solved in some way. 